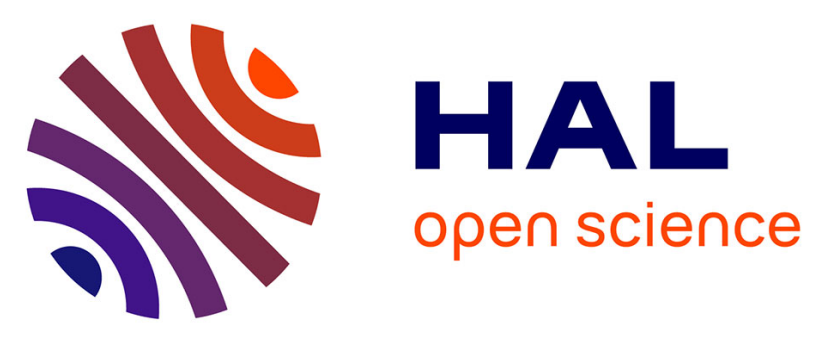

\title{
Résultats du sondage EPTiES (English Pronunciation Teaching in Europe Survey) : l'enseignement de la prononciation dans plusieurs pays européens vu par les enseignants
}

Dan Frost, Alice Henderson

\section{To cite this version:}

Dan Frost, Alice Henderson. Résultats du sondage EPTiES (English Pronunciation Teaching in Europe Survey) : l'enseignement de la prononciation dans plusieurs pays européens vu par les enseignants. Recherche et pratiques pédagogiques en langues de spécialité - Cahiers de l'APLIUT, 2013, Vol. XXXII No 1, pp.92-113. 10.4000/apliut.3586 . hal-01962474

\section{HAL Id: hal-01962474 \\ https://hal.science/hal-01962474}

Submitted on 20 Dec 2018

HAL is a multi-disciplinary open access archive for the deposit and dissemination of scientific research documents, whether they are published or not. The documents may come from teaching and research institutions in France or abroad, or from public or private research centers.
L'archive ouverte pluridisciplinaire HAL, est destinée au dépôt et à la diffusion de documents scientifiques de niveau recherche, publiés ou non, émanant des établissements d'enseignement et de recherche français ou étrangers, des laboratoires publics ou privés. 


\section{Recherche et pratiques pédagogiques en langues de spécialité}

Cahiers de l'Apliut

Vol. XXXII N ${ }^{\circ} 1 \mid 2013$

Les langues de spécialité en Europe Articles

\section{Résultats du sondage EPTiES (English Pronunciation Teaching in Europe Survey) : l'enseignement de la prononciation dans plusieurs pays européens vu par les enseignants}

Results of EPTiES (English Pronunciation Teaching in Europe Survey): A Snapshot of Pronunciation Teaching in Several European Countries, through Teachers' Eyes

Dan Frost et Alice Henderson

p. $92-113$

\section{Résumés}

Français English

Cet article a pour but de présenter certains résultats d'une étude sur l'enseignement de la prononciation dans plusieurs contextes européens ainsi que de discuter des implications de ces résultats. Ce projet est le fruit d'un travail collaboratif entre des enseignants de dix pays européens (Allemagne, Espagne, Finlande, France, Pays-Bas, Irlande, Macédoine, Pologne, Suède et Suisse). Nous présentons ici les résultats d'un questionnaire (843 participants), ainsi que quelques extraits des entretiens semi-structurés que nous avons réalisés. Le questionnaire 
s'appuie sur d'autres travaux menés dans le domaine (Walker 1999 ; Burgess \& Spencer 2000 ; Breitkreutz, Derwing \& Rossiter 2002 ; Macdonald 2002).

Nous nous concentrons dans cet article sur les thèmes suivants : les compétences et la formation des enseignants, ainsi que le niveau et les motivations des apprenants.

The aim of this article is to present certain findings of a study on pronunciation teaching practices in several different European contexts and to discuss some of the implications of these findings. This is a collaborative project between teachers from ten European countries (Finland, France, Germany, Netherlands, Ireland, Macedonia, Poland, Spain, Sweden and Switzerland). In this article, we present some of the results of a questionnaire (843 participants) and some excerpts of the semi-structured follow-up interviews we carried out. The questionnaire builds upon similar studies in this area (Walker 1999; Burgess \& Spencer 2000; Breitkreutz, Derwing \& Rossiter 2002; Macdonald 2002).

In this paper, we are particularly concerned with the following themes: teacher skills and teacher training, learner aspirations and motivations.

\section{Entrées d'index}

Mots-clés : enseignement, Europe, prononciation, phonétique, sondage, entretien, formation en langue

Keywords : Europe, interview, phonetics, survey, teaching, language course, pronunciation

\section{Texte intégral}

\section{Introduction}

Dans le secteur LANSAD en France, l'enseignement de la prononciation est souvent négligé (Diana 2010). La phonétique et la phonologie, souvent confondues avec l'enseignement de la prononciation, sont enseignées dans des facultés de langues, mais sont nettement moins représentées dans les congrès et publications que les trois axes classiques - littérature, civilisation et linguistique. Pourtant, selon une perspective sociolinguistique, l'accent d'un individu sert à identifier son origine ou son identité ethnique/nationale (voir par exemple Giles, Wilson \& Conway 1981; Lindemann 2003; Boyd 2003 ; Munro, Derwing \& Sato 2006). Il est donc fort probable qu'un interlocuteur émette des jugements basés au moins en partie sur la prononciation, jugements très significatifs lorsqu'il s'agit d'une relation entre apprenant et enseignant de langue et où l'expertise perçue de ce dernier est en jeu. Citons par exemple DerivryPlard (2008 : 288) qui déclare « une plus grande compétence linguistique se caractérise par un meilleur accent ou par une meilleure prononciation »1. Pawlak (2008 : 285) attribue également une grande importance à la qualité de l'accent de l'enseignant à cet égard : " un puissant schéma social contribue à mystifier les rôles de locuteur et enseignant, du locuteur natif et de l'enseignant natif $»^{2}$. En partant de ces constats, nous étions curieux de mieux comprendre la situation en France concernant l'enseignement de la prononciation et de voir si elle était semblable à celle d'autres pays européens.

2 Plusieurs sondages concernant l'enseignement de la prononciation ont déjà été menés, mais principalement dans des pays anglophones, tels que le Canada (Foote, Holtby \& Derwing 2011 ; Breitkreutz, Derwing \& Rossiter 2002), l'Australie (Macdonald 2002) et la Grande-Bretagne (Bradford \& Kenworthy 1991 ; Burgess et Spencer 2000). Les études sur les aspects cognitifs, affectifs et conatifs des enseignants d'anglais sont assez rares dans ce domaine en Europe (Walker 1999, en Espagne ; Murphy 2011, en Irlande), et se sont concentrées plutôt sur les apprenants. En Pologne, les chercheurs se sont principalement intéressés à deux questions : d'abord, les relations entre apprenants et le modèle enseigné (Janicka, Kul \& Weckwerth 2005 ; Waniek-Klimczak \& Klimczak 2005) et deuxièmement, le niveau des apprenants par rapport aux modèles choisis (Gonet, Szpyra-Kozłowska \& Święciński 2010 ; Nowacka 2010). Bien que les études citées ci-dessus se concentrent sur le point de vue des apprenants et non sur celui des enseignants, la majorité des sujets se destinent à une carrière d'enseignant 
d'anglais : Gonet, Szpyra-Kozłowska \& Święciński signalent que peu de leurs sujets croient que leur prononciation atteindra le niveau d'un natif. Paunović (2009) décrit un scénario semblable en Serbie. Elle a exploré les interactions entre des critères sociolinguistiques pour tenter d'expliquer les attitudes des enseignants stagiaires envers plusieurs variétés d'anglais. Selon les réponses des participants, la différence la plus significative était celle entre « étranger et incorrect» et « standard et correct ». Les sujets avaient une préférence marquée pour l'anglais britannique ou Received Pronunciation (RP) et pour l'anglais américain ou General American (GA), jugeant les locuteurs natifs qui parlaient une autre variété d'anglais comme des non natifs. En Finlande, peu de recherches existent dans le domaine de l'enseignement de la prononciation, et comme dans les autres pays cités, les chercheurs finlandais se sont surtout intéressés aux apprenants (Lintunen 2004 ; Tergujeff et al. 2011).

Un axe de recherche se concentrant sur l'enseignement de la prononciation dans plusieurs pays européens apparaît donc nécessaire. C'était le but du projet EPTiES (English Pronunciation Teaching in Europe Survey) auquel des enseignants de dix pays d'Europe (Allemagne, Espagne, Finlande, France, Pays-Bas, Irlande, Macédoine, Pologne, Suède et Suisse) ont participé en s'investissant dans la création du sondage dont nous présentons les résultats pour sept pays (tous sauf les Pays-Bas, l'Irlande, et la Suède). Nous présenterons tout d'abord le sondage, puis, dans un deuxième temps, les enseignants, notamment leur niveau et leur formation. Ensuite, nous nous intéresserons aux apprenants, en particulier à leurs compétences et à leurs motivations.

\section{Le sondage}

Le sondage est le résultat d'un effort collaboratif entre dix enseignants provenant d'autant de pays européens. À partir d'une idée lancée lors d'un colloque en décembre 2008, il a fallu presque deux années de correspondance par courrier électronique (avec des documents partagés via Dropbox et/ou Google Docs) pour arriver au sondage final, en demandant conseil à d'autres chercheurs à plusieurs moments critiques : par exemple, un statisticien finlandais est intervenu au moment de la préconception des analyses statistiques. Il s'est avéré trop difficile d'organiser des visio-conférences ; néanmoins, plusieurs membres ont pu se retrouver lors de colloques en juin 2009 et en décembre 2011. Bien que ces interactions collaboratives asynchrones entre autant de personnes puissent ralentir l'avancement d'un projet, les avantages d'une telle approche «lente » dans la génération de savoirs solides sont aussi importants (voir Henderson, à paraître).

5 La version en ligne du sondage a été réalisée et administrée avec LimeSurvey, une application gratuite et open source, et comporte 84 questions divisées en neuf catégories. Cet article se concentre sur deux catégories, celles qui visent la formation des enseignants et leurs opinions et attitudes. Les items comportent des menus déroulants, des échelles Likert, des questions fermées et des questions ouvertes. Certains items du questionnaire reflètent les contextes spécifiques des pays concernés, tels que l'établissement où le sujet enseigné. Un total de 843 personnes ont répondu à ce questionnaire, dont 481 à tous les items, et la majorité des 362 restant à presque tous, une minorité seulement omettant quelques questions ouvertes vers la fin. L'URL du questionnaire a été envoyée aux enseignants des dix pays concernés, dans les secteurs public et privé, en contactant directement les établissements et via les listes de diffusion de groupes professionnels et de sociétés savantes (par exemple, l'APLIUT, l'ARDAA, le GERAS, RANACLES, TESOL-France et l'UPLEGESS pour la France, SUKOL pour la Finlande, ELTAM en Macédoine et ETAS en Suisse). Nous avons également mis les détails du sondage en ligne sur LinguistList et pendant deux ans nous avons distribué des marque-pages promotionnels dans des congrès nationaux et internationaux.

$6 \quad$ Ci-dessous (tableau 1), nous présentons les résultats pour les pays qui comptent au moins 12 questionnaires remplis totalement. 
Tableau 1. Participants par pays, nombre total de participants et nombre de questionnaires remplis à $100 \%$ par pays

\begin{tabular}{|l|c|c|}
\hline Pays & $\begin{array}{l}\text { No de participants } \\
\text { par pays }\end{array}$ & $\begin{array}{l}\mathbf{N}^{\circ} \text { de questionnaires } \\
\text { remplis à 100\% }\end{array}$ \\
\hline Allemagne & 362 & 270 \\
\hline Espagne & 31 & 20 \\
\hline Finlande & 103 & 76 \\
\hline France & $\mathbf{6 5}$ & $\mathbf{5 2}$ \\
\hline Macédoine & 36 & 13 \\
\hline Pologne & 20 & 16 \\
\hline Suisse & 18 & $\mathbf{4 5 9}$ \\
\hline Total & $\mathbf{6 3 5}$ & 12 \\
\hline
\end{tabular}

Pour compléter le questionnaire, nous avons décidé de faire des entretiens semiguidés (Dörnyei \& Taguchi 2010) avec une partie des sujets. Le dernier item du sondage leur proposait de laisser leur adresse courriel s'ils souhaitaient connaître les résultats. Nous avons donc envoyé le premier dépouillement des statistiques à ces personnes, en demandant parallèlement aux répondants français s’ils accepteraient d'être interviewés. Des entretiens avec les personnes ayant répondu par l'affirmative ont ainsi pu être enregistrés avec le logiciel MP3SkypeRecorder. Certains de leurs commentaires seront présentés plus loin dans cet article. Les quatre questions posées, correspondant à des entretiens de 20 à 45 minutes, sont les suivantes :

1. Quelle formation avez-vous reçue au niveau de votre propre prononciation en anglais, ou bien en phonétique et en phonologie, et qu'en pensez-vous ?

2. Quelle formation avez-vous reçue afin d'enseigner la prononciation et/ou la phonétique et la phonologie, et qu'en pensez-vous ?

3. En ce qui concerne la prononciation seulement, qu'enseignez-vous, à quel public et comment?

4. Selon vous, quels sont les défis principaux relatifs à l'enseignement de la prononciation?

8 Concernant les questions du sondage, nous tenons à signaler que, dans le texte du questionnaire, aucune définition n'est fournie pour la notion de " natif », afin d'éviter de biaiser les réponses des participants. Il est néanmoins important de signaler ici le sens que nous attribuons à ce terme. Sans entrer dans le vif du débat complexe (voir Davies 2003) 3 sur les différents facteurs qui ont un impact sur le fait qu'une personne soit qualifiée de locuteur natif ou se qualifie elle-même ainsi, nous soulignons deux critères :

- La priorité au début de la vie : Critère fréquemment mentionné ou sous-jacent aux discussions du grand public ou dans la presse, et traité depuis longtemps par des linguistes tels Bloomfield4.

- La priorité à long terme : la langue dont on se sert le plus et que l'on maîtrise le mieux 5 (Tay 1982). Cela permet au concept de " natif » d'intégrer deux publics parfois ignorés par d'autres définitions : les enfants de parents parlant deux langues différentes ainsi que les adultes qui sont pris pour des natifs, tellement ils maîtrisent une langue apprise après leur jeunesse. 
$9 \quad$ Pour conclure, bien qu'il eût peut-être été judicieux d’inclure dans notre sondage une question ouverte demandant aux participants de définir le terme « natif » ou de leur fournir une définition, nous avons préféré ne pas influer sur les conceptualisations des participants.

\section{Les enseignants}

Dans cette partie, nous verrons tout d'abord quelques données qui nous aideront à comprendre les sujets concernés par ce sondage, afin de mieux interpréter, dans un deuxième temps, les expériences et les opinions des participants sur leur propre prononciation. Enfin, nous nous intéresserons à leur formation à l'enseignement de la prononciation.

11 Le tableau 2 comporte quelques données sur les participants ; les valeurs moyennes sont données pour les sept pays concernés, ainsi que pour la France.

Tableau 2. Quelques données sur les participants

\begin{tabular}{|l|l|l|}
\hline & Données (globales) & Données (France) \\
\hline Sexe & $77 \%$ femmes & $75 \%$ femmes \\
\hline Âge moyen & 42,95 ans & 46 ans \\
\hline Natif ou non natif & $88,21 \%$ non natifs & $74 \%$ non natifs \\
\hline Enseignement public ou privé & $88,21 \%$ public & $97 \%$ public \\
\hline
\end{tabular}

La majorité des participants sont des femmes (77\%), mais il existe des écarts importants : jusqu'à 95,1 \% de femmes en Finlande et 92,3\% en Macédoine, tandis qu'en Espagne elles ne représentent que $35 \%$ des sondés.

L'âge moyen des participants les plus jeunes était celui des enseignants polonais et macédoniens, 24 et 29 ans respectivement, bien en-dessous de la moyenne générale (42,95 ans). En effet, la majorité des participants de Pologne avaient la possibilité de donner des cours alors qu'ils étaient en Master, thèse, ou bien débutaient tout juste leur carrière d'enseignant. L'âge moyen des Français (46 ans) est légèrement au-dessus de la moyenne, ce qui semble normal car il faut souvent du temps pour devenir un enseignant certifié et expérimenté ${ }^{6}$. Bien que l'âge moyen en Suisse soit semblable à celui de la France, les enseignants ayant répondu au questionnaire en France avaient en moyenne 21 ans d'expérience du métier, contre seulement 15 ans en Suisse. En France, les enseignants choisiraient leur métier assez tôt dans leurs études, tandis qu'en Suisse, l'enseignement n'est peut-être pas le premier choix de carrière.

Quant à l'origine des participants, sur les sept pays, presque neuf personnes sur dix disent que l'anglais n'est pas leur langue maternelle $(88,21 \%)$. En Pologne et en Macédoine, $100 \%$ des sujets sont des non natifs, et en Allemagne $96 \%$. En revanche, en Suisse, 84 \% des sujets sont des anglophones natifs ; en France, pays voisin, 74 \% des participants sont des anglophones non natifs. Afin de vérifier si ces statistiques se confirment au niveau de la population générale d'enseignants, nous avons cherché des chiffres nationaux pour ces pays, en vain. En ce qui concerne le niveau d'éducation, dans deux pays seulement les sujets se disent titulaires d'une qualification universitaire spécifiquement destinée à l'enseignement de l'anglais comme langue étrangère. D'abord, en Suisse, 13 répondants sur 17 ont un diplôme spécifique (DipTEFL, CELTA, MEd TESOL, etc.). En Finlande, la majorité des sujets sont titulaires d'au moins un Master en anglais avec une formation professionnelle comme partie intégrante du programme. Ensuite, les jeunes participants polonais et macédoniens étaient pour la plupart des étudiants en Master ou en thèse. En Espagne, tous sauf un avaient une licence, et 25 \% avaient un Master ou une thèse. En France, plus de la moitié des sujets étaient certifiés et/ou agrégés7. 
Les participants enseignent principalement dans des établissements du secteur public (92,2 \% en Finlande, 93,93 \% en Allemagne, 80,65 \% en Espagne), sauf en Macédoine, où $76,92 \%$ des participants enseignent dans le privé. Les Polonais enseignent dans le secteur public, mais donnent souvent des cours dans des écoles privées le soir et le week-end. Les participants suisses enseignent principalement aux adultes $(76,9 \%)$ ou dans des collèges et lycées (21,5\%). Les Finlandais se répartissent surtout entre l'école primaire $(29,1 \%)$, le collège $(31,1 \%)$ et le lycée $(27,2 \%)$, avec seulement quelques participants travaillant à l'université. Les trois-quarts des participants en Allemagne enseignent dans des écoles secondaires : (40,50 \%) au Gymnasium (10 à 18 ans), 20,94 \% dans une Realschule (10 à 16 ans) et 16,25\% dans une Grundschule (6 à 10 ans). Enfin, si autant de participants enseignent dans le public, c'est fort probablement que nous n'avons pas trouvé de moyen efficace pour contacter les enseignants du secteur privé. Si l'on prend le cas français, à part TESOLFrance, il n'existe pas de réseau qui permette de contacter des enseignants libéraux, auto-entrepreneurs, vacataires, etc.

Nous voulions également creuser la question du statut de la prononciation et de l'anglais, en lien avec l'auto-évaluation de la prononciation des enseignants. Dans ce but, un item du questionnaire portait sur l'importance de l'anglais par rapport à d'autres langues (item 60), et un autre sur l'importance de sa prononciation par rapport aux autres compétences (item 61). Les moyennes dans les deux cas étaient plutôt élevées, de 4,66 et de 3,77 respectivement sur 5 (5 étant « extrêmement important »). Un troisième item portait sur l'auto-évaluation de la prononciation des participants : la valeur moyenne était de 4,17 (5 étant « natif »). Dans cette section, nous allons analyser les réponses à ces items, présentées dans les graphiques des figures 1, 2 et 3 .

Fig. 1. Réponses à l'item 60 (l'importance de l'anglais par rapport à d'autres langues)

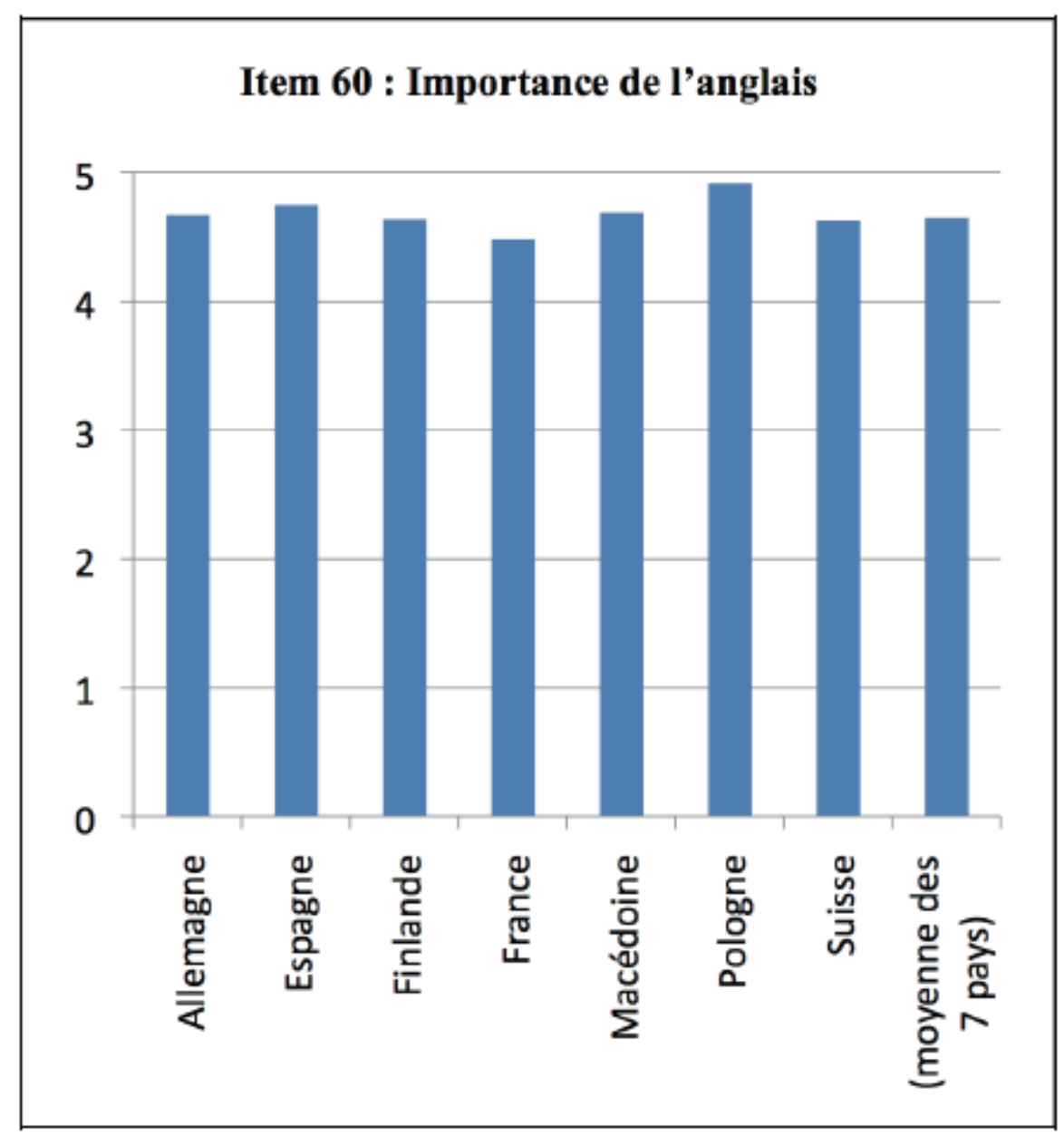

$1=$ « pas important du tout $», 5=$ « très important »

Les chiffres en faveur de l'importance de l'anglais sont élevés dans tous les pays concernés, avec une moyenne de 4,65 sur 5. Le score le plus bas revient à la France 
$(4,48)$ et le plus élevé à la Pologne $(4,92)$ et c'est à la lumière des contextes finlandais et macédoniens qu'une explication de cette attitude pourrait se trouver. En Finlande, l'importance de l'anglais par rapport aux autres langues est élevée $(4,64)$ et les participants ont souvent mentionné leur utilisation régulière de l'anglais avec des collègues, des amis, etc. Cependant, les Finlandais sont très préoccupés par l'hégémonie de l'anglais : un participant a écrit que l'anglais « n'est pas la seule langue que l'on devrait apprendre ». Il est vrai que le gouvernement finlandais a toujours investi beaucoup de ressources dans une palette plus large de langues, en particulier l'allemand, le français et le russe, mais les jeunes Finlandais semblent moins intéressés par ces autres langues (Kangasvieri et al. 2011 ; Sajavaara, Luukka \& Pöyhönen 2007). Les résultats macédoniens sont très semblables $(4,69 / 5)$ et l'importance économique de l'anglais ainsi que son statut de « langue mondiale » ont été cités plusieurs fois dans les commentaires.

Fig. 2. Réponses à l'item 61 (l'importance de la prononciation par rapport à d'autres compétences langagières)

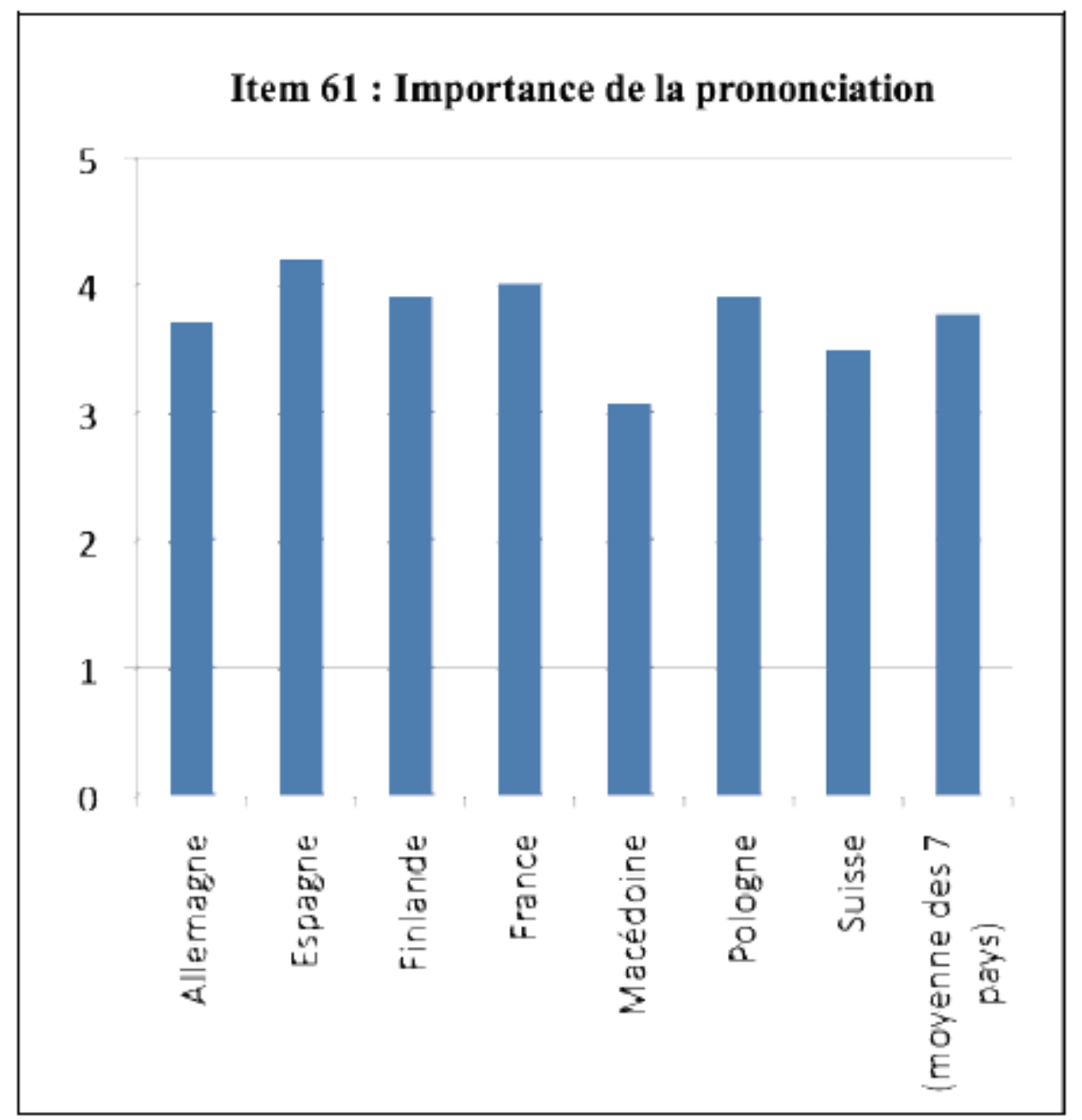

$1=$ « pas important du tout », $5=$ « très important »

Les réponses à l'item 61 (fig. 2), qui évoque l'importance de la prononciation de l'anglais par rapport à d'autres compétences langagières, renforcent et approfondissent ces notions. Les résultats allemands $(3,72)$ soulignent le changement qui s'est opéré dans le statut de l'anglais dans les écoles primaires et secondaires à l'instar de la politique nationale et européenne. Selon Hilgendorf (2007 : 144), l'anglais n'est plus une langue étrangère, mais une langue "supplémentaire ». Cela pourrait également expliquer le score obtenu en Suisse (3,5), un pays à politique linguistique plurilingue où le statut officiel de l'anglais fait souvent débat. La moyenne la plus élevée est en Espagne $(4,2)$, où le besoin d'améliorer à la fois la prononciation des apprenants espagnols et la formation des enseignants dans ce domaine est attesté dans la littérature (Donovan 2001 ; Levey 1999, 2001 ; Pavon 2001 ; Pavon \& Rosado 2003). Malheureusement, comme de nombreux commentaires des participants exerçant en 
Espagne l'ont démontré, les exigences du système scolaire espagnol sont telles que la nécessité de préparer des examens écrits prime souvent sur un travail poussé de la prononciation. Si, pour l'ensemble des pays, un pourcentage non-négligeable (19,2 \%) des sujets a attribué $5 / 5$ à la prononciation en anglais (" extrêmement important »), la majorité s'est montrée assez pragmatique, lui attribuant 4/5 (52,6 \%) et 3/5 (26,9\%). Une fois de plus les raisons invoquées relèvent des motivations extrinsèques, telles que la place de l'anglais dans le monde du commerce international. Les enseignants semblent reconnaitre le désir de leurs apprenants d'apprendre l'anglais pour communiquer, donc la prononciation est certes importante, mais dans le sens où une maîtrise minimale est requise pour la communication. Une telle focalisation sur la communication pourrait expliquer les deux scores les plus bas (Suisse 3,5; Macédoine 3,08). Ce dernier pays, indépendant seulement depuis 1991, est actuellement candidat à l'adhésion à l'Union Européenne. Sa réussite sur la scène internationale passe obligatoirement par la maîtrise des langues étrangères.

Nous nous sommes ensuite intéressés au niveau de prononciation des enseignants, ou plutôt à leur perception de leur propre niveau (fig. 3). D'abord, il faut constater que la moyenne $(4,18)$ reste assez élevée et que celle attribuée par les participants en France et en Suisse $(4,1)$ en est très proche. En ce qui concerne les valeurs les moins élevées, les participants exerçant en Pologne portent un regard assez modeste sur leurs compétences : 3,92 , score similaire à celui des allemands; le commentaire suivant montre en revanche que certains d'entre eux mettent la barre assez haut : " Je suis capable de ne pas avoir l'accent allemand, donc les élèves ne savent pas d'où je viens, mais ils sont certainement au courant que je ne suis pas natif ». Le score le plus élevé fut attribué par les enseignants en Macédoine $(4,54)$, dépassant de peu celui de l'Espagne $(4,25)$ et de la Finlande $(4,24)$; pour le moment, nous ne saurions expliquer ces scores, même à la lumière des commentaires.

Fig. 3. Réponses à l’item 63 (Comment estimez-vous votre propre prononciation ?)

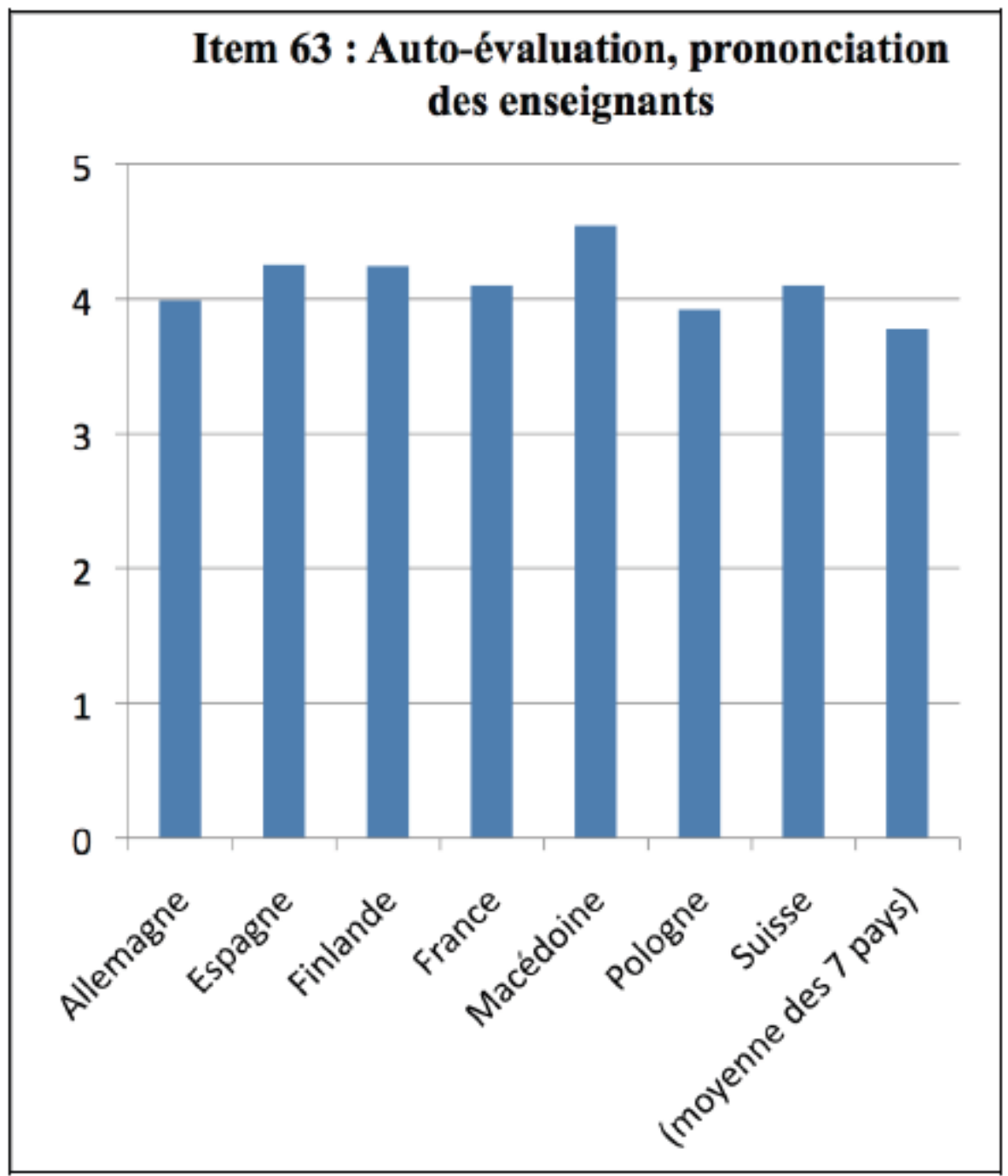


1 = « extrêmement mauvaise », 5 = « excellente »

Pour terminer cette partie sur les enseignants, passons à la formation qu'ils ont reçue pour enseigner la prononciation. Les trois items concernant ce domaine étaient les suivants :

57. En ce qui concerne la prononciation, veuillez attribuer une note sur 5 à la formation que vous avez reçue, 1 étant « très mauvaise » et 5 étant « excellente ». 58. Veuillez nous dire quelle quantité de formation spécifique à l'enseignement de la prononciation vous avez reçue. Vous pouvez mentionner le nombre d'heures, de semaines, de mois, d'années, etc.

59. Veuillez expliquer le contenu et/ou le style de la formation que vous avez reçue pour enseigner la prononciation. Vous pouvez mentionner des stages, des méthodes utilisées, etc.

Ces trois items étaient précédés d'un rappel expliquant qu'il ne s'agissait pas de la formation à l'enseignement de l'anglais en général, mais de la formation spécifique à l'enseignement de la prononciation. En dépit de cette précision, de nombreux candidats ont cité des exemples issus de leur formation générale ou bien de l'enseignement reçu quand ils étaient eux-mêmes étudiants en premier cycle universitaire. Il y a donc souvent confusion entre phonétique et prononciation et entre formation professionnelle et formation initiale. Cette double confusion est très fréquente car, comme les réponses aux items 58 et 59 le démontrent, la majorité des participants ont reçu tellement peu de formation professionnelle spécifique qu'ils se sont appuyés sur la seule expérience qu'ils avaient, c'est-à-dire celle d'un étudiant de première année dans un département de langues universitaire européen.

Fig. 4. Réponses à l'item 57 (la qualité de la formation en prononciation reçue)

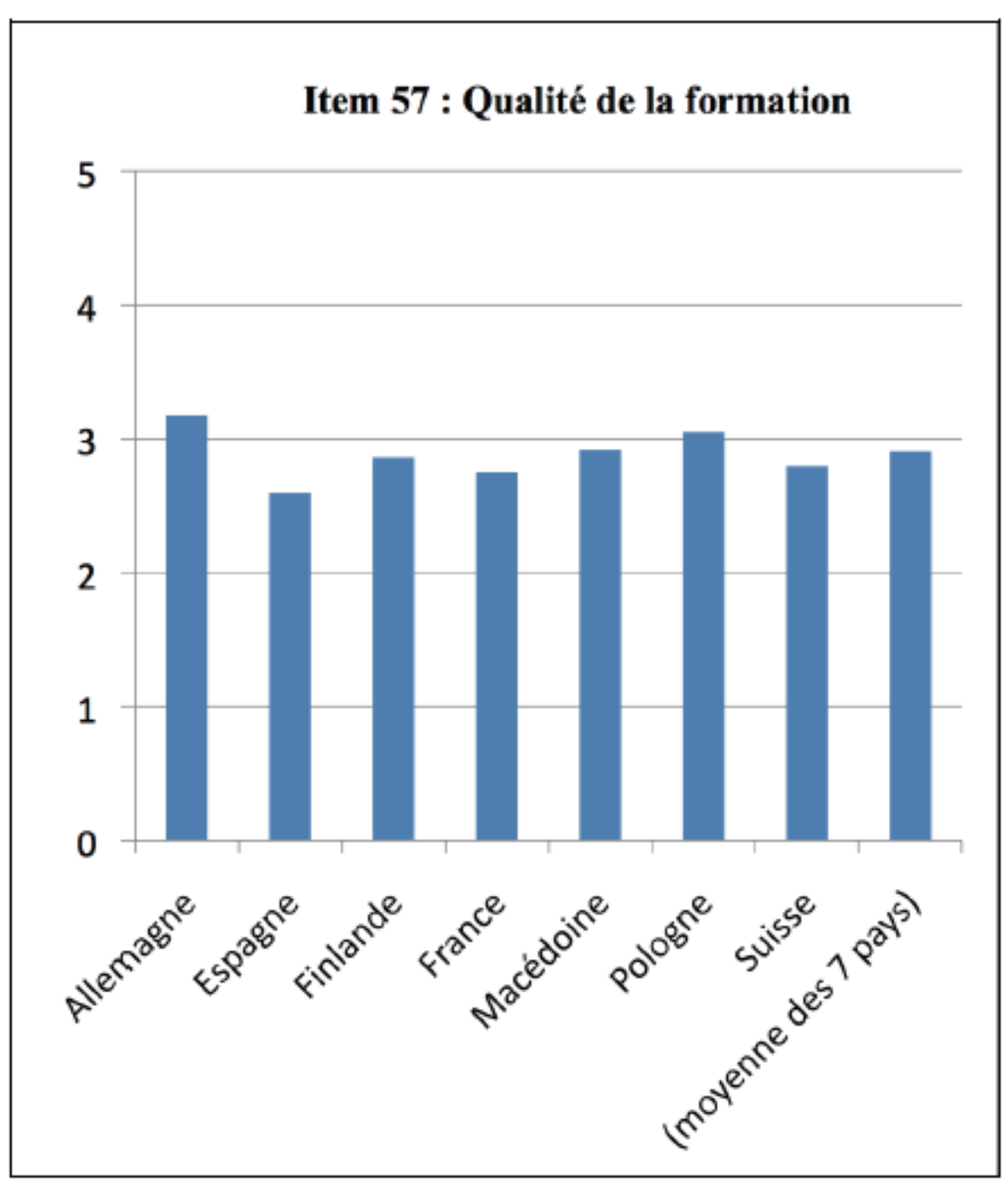

$1=$ « très mauvaise » et $5=$ « excellente » 
En effet, la moyenne des réponses à l'item $57(2,91)$ est nettement inférieure aux moyennes pour les items 61 et 63 présentées ci-dessus. Pour rappel, les participants estiment que la prononciation est une compétence importante (4,65 sur 5) et que leur propre prononciation est plutôt bonne (4,18 sur 5). La comparaison de ces deux moyennes au-dessus de 4 et celle de 2,91 est révélatrice, car même s'il n'y a pas eu confusion entre phonétique et prononciation et entre formation initiale et professionnelle, il semble probable qu'en ce qui concerne l'enseignement de la prononciation au moins, les enseignants qui ont participé à cette étude sont des amateurs. Par " amateurs », nous entendons que les participants sont souvent passionnés par le sujet (du latin amator), mais aussi qu'ils ont reçu peu ou pas de formation professionnelle. Malgré ce manque, ils reconnaissent l'importance de cette compétence et ils ont souvent acquis leur expertise autrement.

Le contexte français de la formation des enseignants à l'enseignement de la prononciation est peut-être le plus dramatique des pays concernés par cette étude. Sur 65 sujets exerçant en France ayant répondu à ces trois items, 19 ont répondu qu'ils avaient reçu peu ou pas de formation, 19 ont seulement mentionné les cours de phonétique qu'ils ont reçus en Licence, et 9 ont mentionné des séminaires ou des ateliers auxquels ils ont assisté indépendamment de leur formation à l'IUFM. De même que pour les sujets exerçant dans les autres pays, les cours à l'université en premier cycle semblent avoir été très traditionnels, consistant essentiellement en cours magistraux accompagnés de travaux dirigés ou de travaux pratiques, parfois en laboratoire de langues. En fait, très peu de sujets français ont eu quelque chose de positif à dire à ce sujet. Au mieux, on peut lire par exemple : « on a eu quelques cours sur la prononciation de l'anglais, l'intonation, etc., mais uniquement la théorie et aucune démonstration sur comment les enseigner ». Et comme un répondant l'a expliqué, « connaître un sujet n'est pas la même chose que l'enseigner ».

Les entretiens semi-structurés ont renforcé l'impression donnée par les réponses aux items 57 à 59, à savoir un manque quasi-total de formation professionnelle en France à l'enseignement de la prononciation. Les cours de préparation aux concours de recrutement des professeurs de l'enseignement secondaire ainsi que les cours à l'IUFM, selon nos sujets, n'ont que très peu traité de la prononciation. Le commentaire suivant est loin d'être unique pour le contexte français : " Il n'y a eu aucune mention, à toute étape du processus, ni de la prononciation ni de la phonétique ». Une des répondantes a même mentionné un « cercle vicieux » en ce qui la concernait : comme elle n'avait pas reçu de formation spécifique, elle avait « esquivé » la prononciation pendant toute sa carrière. Une autre a écrit qu'elle avait l'impression « d'errer dans le noir ». Enfin, ces entretiens nous ont donné l'occasion d'explorer la réticence de certains sujets à enseigner la prononciation.

Mis à part le manque de formation en général, les entretiens avec certains participants français nous ont apporté deux précisions. Premièrement, nombreux sont ceux qui croient que l'enseignant doit « maîtriser » l'alphabet phonétique international (API) pour enseigner la prononciation. En réalité, bien que ces connaissances puissent s'avérer utiles, on peut travailler efficacement la prononciation sans avoir recours à l'API ${ }^{8}$. Deuxièmement, plusieurs sujets semblent convaincus que l'enseignement de la prononciation nécessite un recours à la technologie et, parfois, ils attribuent leur réticence à enseigner la prononciation à un manque de compétences technologiques. Par « technologie », les sujets entendent une variété d'outils, allant du laboratoire de langues audio ou multimédia à des logiciels dédiés en passant par de nombreux dispositifs sur Internet. Encore une fois, à notre avis ces technologies peuvent être un complément utile à l'enseignement traditionnel, mais dans le domaine de la prononciation comme dans d'autres domaines de l'enseignement des langues, le recours à la technologie n'est bien évidemment pas obligatoire et ne devrait pas démotiver les enseignants.

En Allemagne, les enseignants étaient également peu satisfaits de leur formation à cet égard $(2,86)$. À part les nombreux commentaires qui témoignent de la même pénurie qu'en France, ou ailleurs, un thème est récurrent, manifeste dans le commentaire suivant : " les professeurs à l'université et les formateurs des enseignants présument que si on parle correctement la langue, on sera capable de transmettre ces 
compétences (je ne sais comment) aux enfants ». En Pologne, peu de sujets (18,75 \%) ont déclaré avoir reçu une formation spécifique pour enseigner la prononciation. En Espagne, la formation se limitait essentiellement à des modules d'un an ou deux à l'université de 30 à 60 heures typiquement, mais ici aussi, il semble qu'il s'agisse de cours de phonétique. Seulement $10 \%$ des sujets espagnols avaient suivi des stages après l'université, mais $27,77 \%$ avaient reçu peu ou pas de formation et $27,77 \%$ se décrivent comme autodidactes dans la matière. Les Macédoniens ont reçu pour la plupart une formation traditionnelle universitaire en phonétique, mais peu de formation professionnelle. Le commentaire suivant de la part d'un sujet macédonien démontre que, même si les enseignants ont bien travaillé sur leur propre prononciation, certains ont conscience que cela ne suffit pas : « Je crois que les enseignants devraient être très bien formés pour pouvoir bien enseigner la prononciation à leur tour ».

Seuls les Espagnols $(3,05)$ et les Finlandais ont attribué une note au-dessus de 3 $(3,18)$ pour la qualité de leur formation à l'enseignement de la prononciation. Presque la moitié des participants finlandais ont décrit un ou plusieurs stages professionnels et il n'y a pas eu cette fois la double confusion entre phonétique et prononciation et entre formation professionnelle et formation initiale, qui était manifeste dans les autres pays, notamment en France. Les sujets suisses témoignent d'une grande variété au niveau de la quantité de formation, les réponses allant de " rien du tout » à des références aux stages effectués pendant la préparation au CELTA 9 ou à des modules spécifiques pendant le Bachelor's ou bien le PGCE ${ }^{10}$. La Suisse est un des seuls pays où la formation initiale et la formation continue des enseignants incluent du contenu spécifique à la prononciation. Dans les commentaires, nous pouvons lire, par exemple : « nous avons regardé d'autres enseignants sur des DVD » ou bien « un intervenant nous a montré des techniques que nous avons ensuite essayées et puis nous en avons parlé en petits groupes ». Comme dans tous les autres pays, plusieurs commentaires sur la nature autodidacte des compétences des sujets en Suisse sont également présents, mais les enseignants de ce pays ont pu, à plusieurs reprises, faire référence à des auteurs ou à des ouvrages spécifiques.

28 Pour conclure cette partie, il semble que la norme soit peu ou pas du tout de formation professionnelle spécifique à l'enseignement de la prononciation, que les enseignants non natifs s'appuieraient surtout sur la formation en phonétique qu'ils ont reçue en premier cycle, et que nombreux sont ceux qui se sont auto-formés. La situation est très difficile à résumer pour les natifs, qui ont des parcours très variés, mais, le plus souvent, ils sont également peu ou pas formés dans ce domaine.

\section{Les apprenants}

Cette partie aborde les perceptions des participants sur les motivations et les compétences de leurs apprenants. Les items concernés sont les suivants :

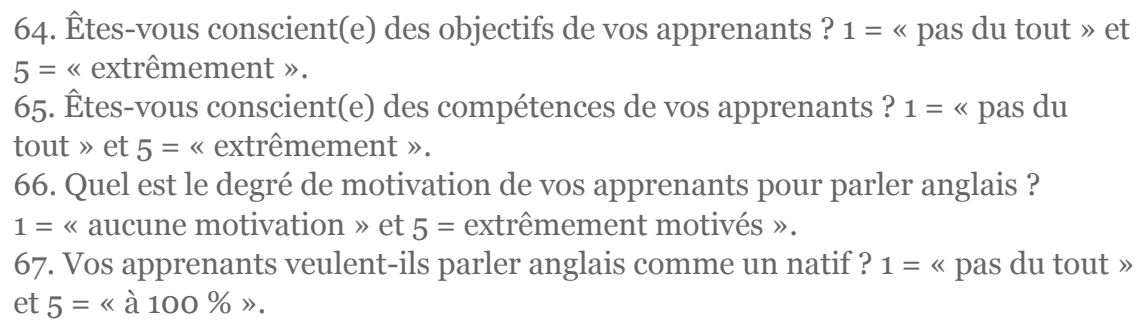

30 Ces résultats sont à interpréter avec soin : non seulement les données ne concernent que les perceptions des enseignants, mais les réponses à ces items sont particulièrement influencées par des facteurs culturels et les contextes d'apprentissage dans chaque pays.

Fig. 5. Réponses à l'item 64 sur la conscience des objectifs des apprenants 


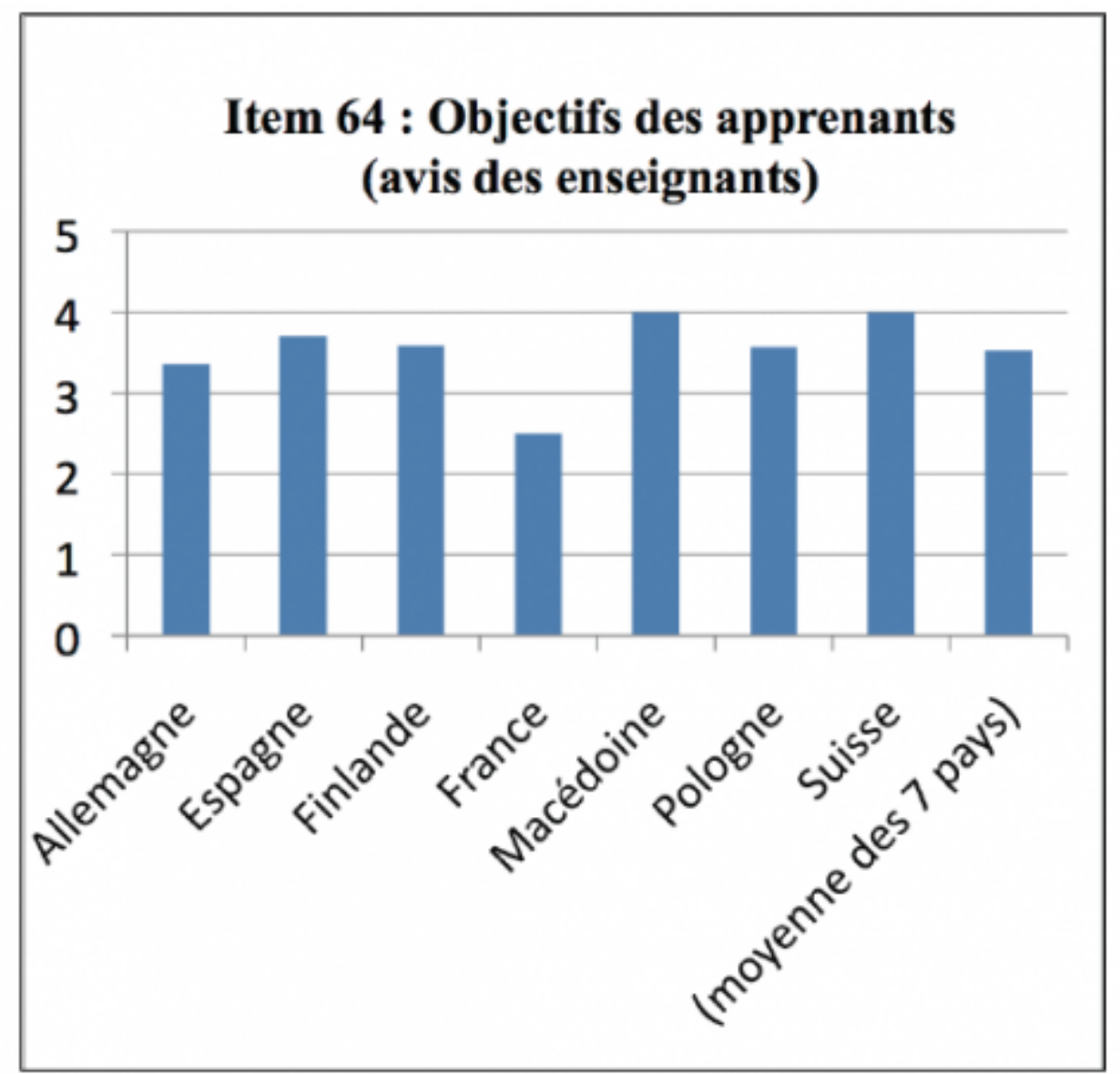

Fig. 6. Réponses à l'item 65 sur la conscience des compétences des apprenants

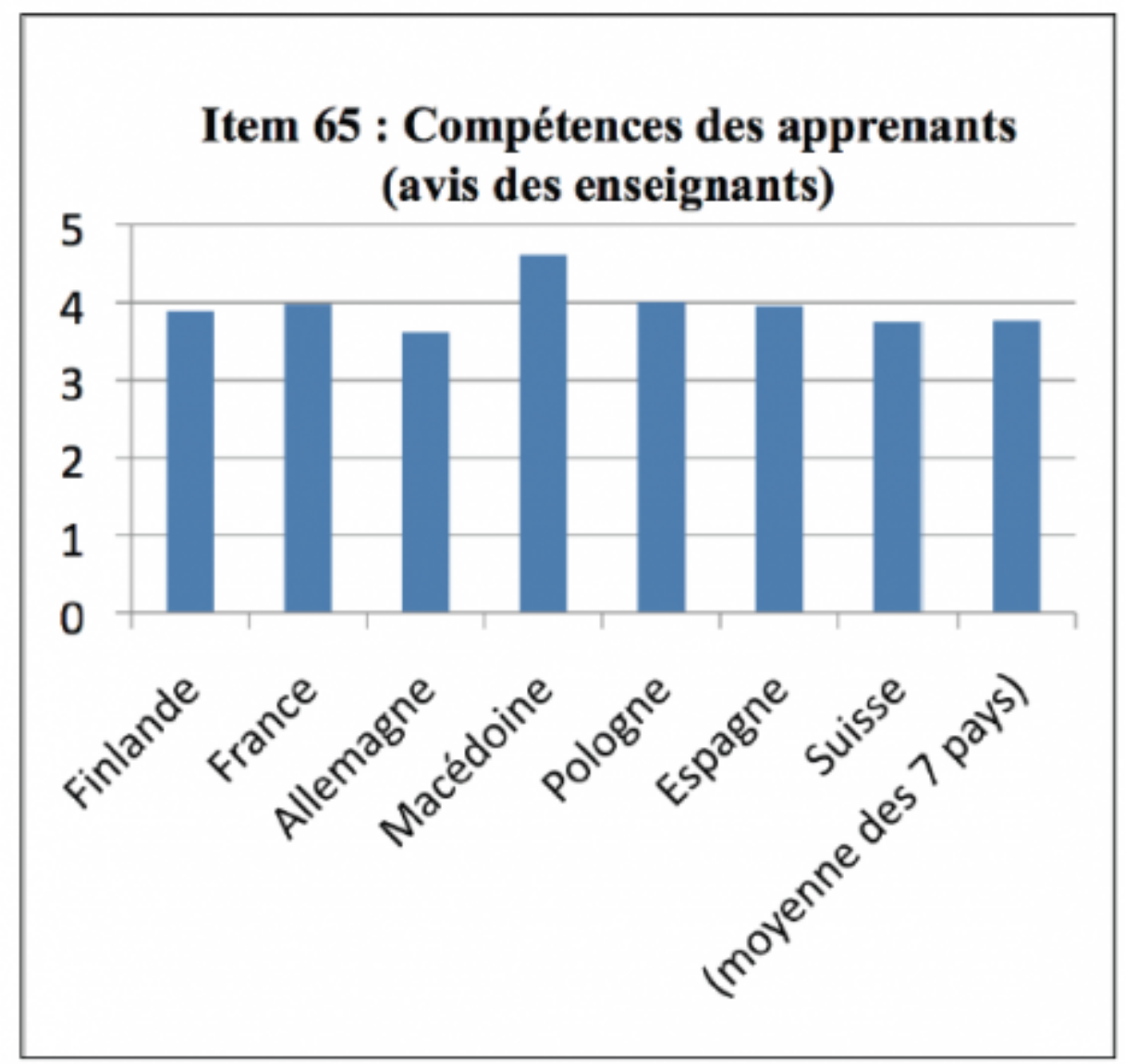

Sur les deux diagrammes des figures 5 et 6 , il semble que, de manière générale, les enseignants se pensent légèrement plus conscients des compétences de leurs apprenants (une moyenne de 3,77) que de leurs objectifs (3,53). Ceci est plus net en Pologne (une moyenne de 4/5 pour les compétences et de 3,57/5 pour les objectifs). Les 
résultats en Finlande vont dans le même sens. Le fait que de nombreux participants finlandais soient issus du primaire $(29,1 \%)$ et des collèges $(31,1 \%)$ explique peut-être une des remarques faites : " je sais ce que leurs objectifs devraient être!». Seuls les enseignants en Suisse prétendent être moins au courant des compétences (3,75/5) que des objectifs $(4 / 5)$ de leurs étudiants. Par rapport à la conscience des objectifs des apprenants, le score le plus bas $(2,5)$ est en France. Ceci est peut-être dû au fait que la majorité des participants exercent dans l'éducation supérieure, souvent en secteur LANSAD. Dans certaines universités, une classe peut compter jusqu'à 50 étudiants et les relations entre étudiants et enseignants sont traditionnellement assez distantes. Ces facteurs ne sont pas, bien évidemment, propices à des discussions entre l'enseignant et ses apprenants sur des sujets tels que la motivation.

Fig. 7. Réponses à l'item 66 sur le degré de motivation des apprenants pour parler anglais

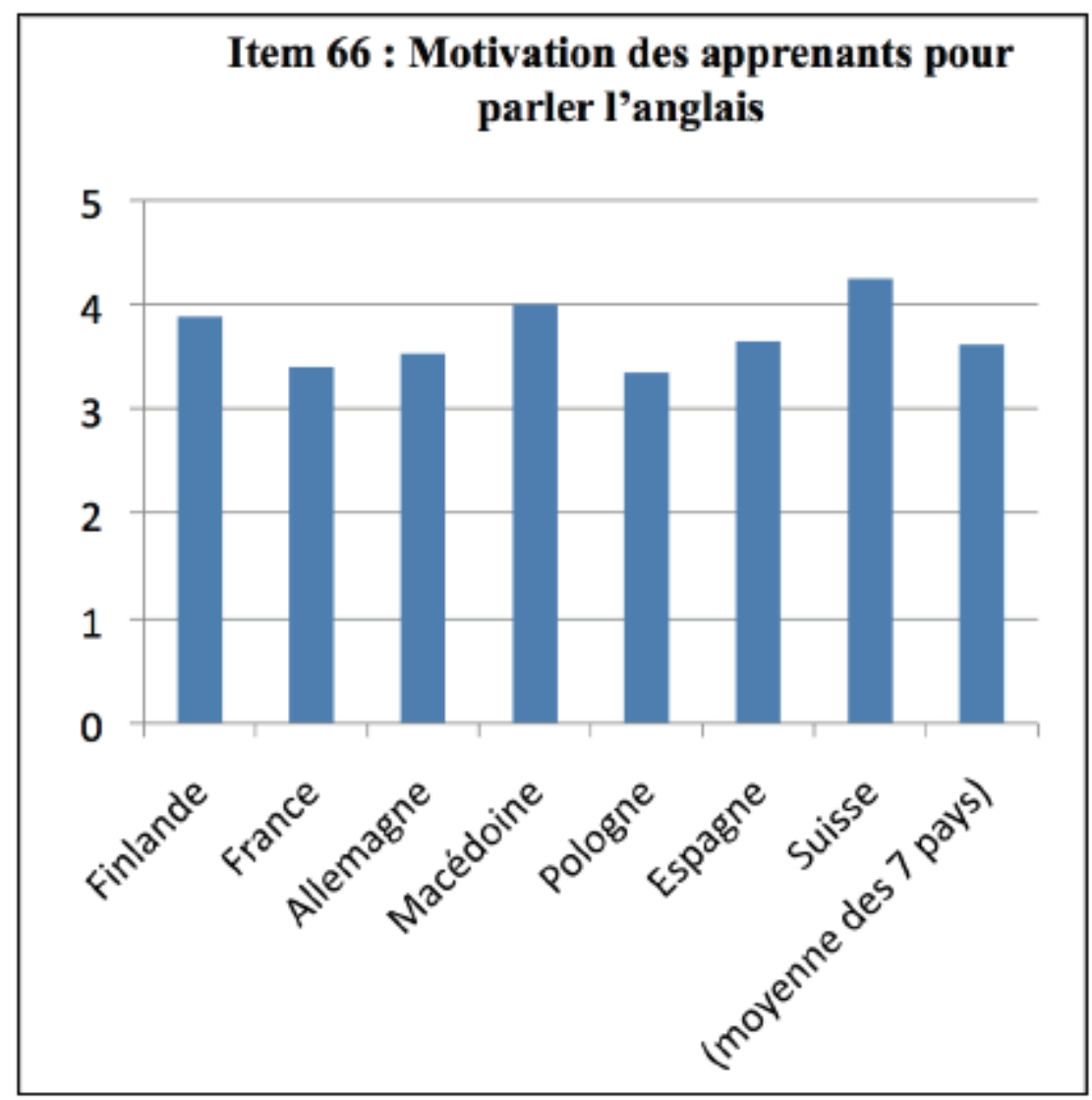

Fig. 8. Réponses à l'item 67 sur le désir des apprenants de parler anglais comme un natif 


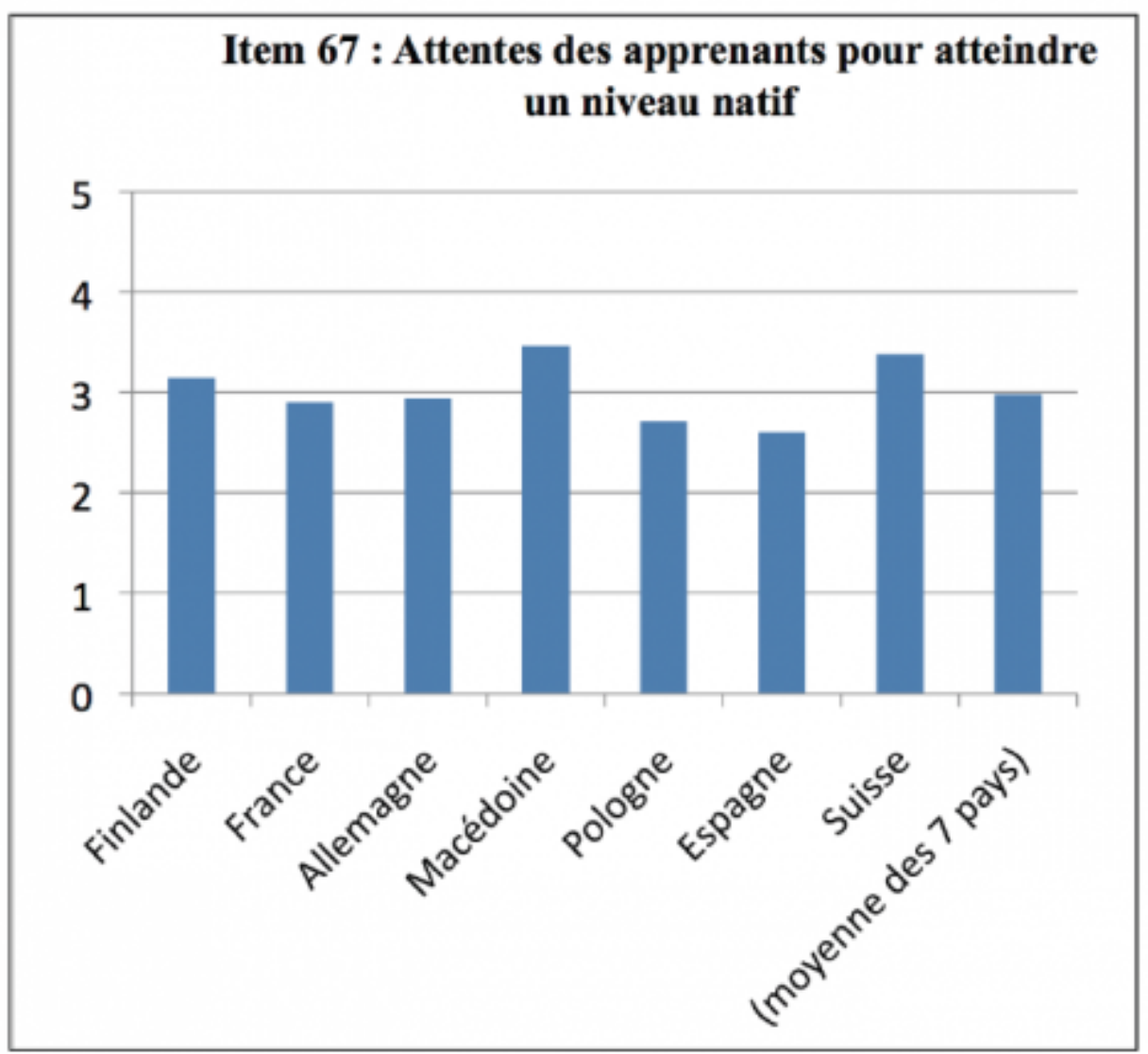

Les deux graphiques (figures 7 et 8) représentent les items sur la motivation des apprenants pour apprendre l'anglais et pour parler anglais comme un natif. Les scores sont systématiquement plus élevés pour la première question que pour la deuxième. Si nous regardons plus en détail, nous pouvons voir que les participants suisses attribuent le score le plus élevé $(4,25)$ à la motivation de leurs apprenants pour apprendre l'anglais, ce qui refléterait l'importance de l'anglais parmi les chercheurs suisses ${ }^{11}$, ainsi que la politique plurilingue que nous avons évoquée plus haut (voir Dürmüller 2002). Par rapport à la prononciation native, de nombreux cours en master sont dispensés en anglais dans plusieurs filières et une prononciation native n'y est bien sûr pas requise. Cependant, en Suisse, le score pour cet item est presque le plus élevé $(3,38)$ juste derrière celui de la Macédoine $(3,46)$, les deux pays étant au-dessus de la moyenne $(2,98)$. En Pologne, les attentes peu élevées $(2,71)$ s'expliquent peut-être par le fait que la majorité des participants polonais enseignent l'anglais aux jeunes enfants. Les Espagnols affichent le score le moins élevé $(2,6)$ de tous.

Quant au contexte français, il semblerait - selon les participants au sondage - que les apprenants soient aussi motivés pour apprendre à parler l'anglais $(3,4)$ que les Polonais $(3,35)$. Ce chiffre est à contraster avec l'importance de l'anglais par rapport à d'autres langues de l'item 6o, évalué par des participants français à 4,48 sur 5 et à 4,92 par les Polonais, au-dessus de la moyenne (3,78). Peut-être l'importance de bien parler l'anglais est-elle vue plutôt par rapport aux autres compétences (lecture, écriture, compréhension orale). Les sujets français estiment également qu'il n'y a que relativement peu d'espoir de parler l'anglais comme un natif $(2,9)$, un score étonnamment semblable à celui des participants allemands $(2,94)$, tout en étant plus élevé que celui des Espagnols $(2,6)$ ou des Polonais $(2,71)$. Afin de mieux comprendre les chiffres français, regardons le contexte d'un grand nombre d'apprenants concernés par cette étude. En effet, dans le secteur LANSAD en France, le niveau de motivation et les attentes des étudiants sont souvent plus bas qu'ils ne le sont dans leur matière principale (Taillefer 2002). Ensuite, bien que le vocabulaire de l'anglais et du français soient souvent très semblables (et sans vouloir nier les différences entre l'anglais et les autres langues concernées par ce travail), ne perdons pas de vue les différences phonologiques entre ces deux langues (Cruttenden 2001; Swan \& Smith 2001; Hirst \& Di Cristo 1998 ; Blum 1999 ; Frost 2011). Ce fait linguistique rend la tâche de l'apprentissage du système phonologique de l'anglais très difficile pour les apprenants, 
et encore plus pour ceux du secteur LANSAD. Enfin, avec un soupçon d'ironie, un commentaire finlandais résume bien un point de vue supposément répandu en LANSAD, selon lequel il n'est pas nécessaire de maitriser parfaitement la prononciation de la langue cible pour s'en servir dans son métier : "Les pilotes de Formule 1 finlandais ont montré qu'il n'est pas nécessaire de prononcer parfaitement l'anglais pour devenir riche et célèbre ».

\section{Conclusion}

Nous nous sommes focalisés dans cet article sur les compétences et la formation des enseignants, ainsi que sur les aspirations des apprenants.

Tout d'abord, les enseignants ne sont pas satisfaits de leur formation à l'enseignement de la prononciation. Cependant, puisqu'elle est perçue comme étant essentielle à la maitrise d'une langue, ils tentent par divers moyens complémentaires d'acquérir les compétences nécessaires. Cette situation pose la question de savoir si l'on devrait accepter que la formation professionnelle des enseignants néglige autant cet aspect indispensable à l'apprentissage d'une langue étrangère. Il serait inadmissible par exemple, pour un enseignant, de proclamer qu'il trouve difficile la grammaire et de ce fait, de ne pas l'aborder avec les apprenants. L'Europe se trouverait donc dans une situation semblable à celle des États-Unis dans les années 1990, quand moins de la moitié des programmes de Master en TESOL proposaient un module de phonologie (Murphy, J.M. 1997). Ceci est en contradiction directe avec les certifications en place au niveau européen et international. D'abord, à l'intérieur de ses frontières, l'Europe s'aligne maintenant sur le CECR, avec l'objectif de " Maitriser le système phonologique » détaillé dans la catégorie « Compétence de communication langagière » (Conseil de l'Europe 2001). Ensuite, les certifications internationales telles que IELTS, TOEFL et TOEIC sont passées chaque année par des milliers d'étudiants européens, afin de faire avancer leurs projets professionnels ou de rendre possible une mobilité scolaire. L'évaluation de la prononciation fait partie intégrante de ces examens payants. Il apparaît donc évident à la lumière de ce sondage que nos apprenants ont besoin d'un enseignement de la prononciation de qualité pour leur réussite scolaire et professionnelle, enseignement que la formation de leurs enseignants ne favorise pas. Il nous faudra capitaliser sur le fait que, selon les dires des participants au sondage, nos apprenants semblent plutôt pragmatiques et paraissent motivés pour l'apprentissage de l'anglais mais pas focalisés sur l'idée de parler comme un natif.

Les perspectives de recherches et d'actions ne manquent pas. Par-dessus tout, nous aimerions utiliser cette expérience collaborative pour contrer ce que nous ne pouvons qu'appeler un cercle vicieux. Les participants en France (74 \% anglophones non natifs) ont évalué leur propre prononciation plutôt favorablement $(4,1 / 5)$, moyenne identique à celle de la Suisse où $84 \%$ des participants sont des anglophones natifs. Cependant, dans les entretiens, un manque d'assurance de la part des enseignants français se manifeste par rapport soit à leur propre prononciation, soit à leur façon de l'enseigner (par exemple un souci révélé dans les commentaires sur la maîtrise de l'API et/ou de diverses technologies). Une enseignante en France indique que les grands défis dans l'enseignement de la prononciation sont au nombre de deux : aider l'apprenant à améliorer sa prononciation afin de ne pas en avoir honte et rendre les locuteurs non natifs facilement compréhensibles. Cependant, plusieurs personnes interviewées avouent se sentir mal à l'aise face à ces gageures, en partie car ils confondent phonétique et prononciation : "Je suis tout à fait consciente du fait que je ne sais strictement rien de la phonétique $»^{12}$. Nombreux sont ceux qui ont insisté sur le fait que ce ne sont pas les apprenants mais les enseignants qui sont réticents, et cette hésitation, voire cette réticence, est à l'origine du problème. Une stratégie d'évitement semble donc mise en place et l'enseignant ou le formateur finalement n'enseigne que peu ou pas du tout la prononciation. Si la prononciation n'est pas enseignée, elle peine à s'améliorer chez l'enseignant comme chez l'apprenant, et les deux défis ne seront pas relevés. On en 
arrive à la situation actuelle, où, selon un interviewé : « Une mauvaise prononciation est chronique dans l'ensemble du système éducatif français $»^{13}$.

Il faut tenter de briser ce cercle vicieux, tout en conjurant les peurs. Évidemment, la recherche et la formation professionnelle ont leur rôle à jouer dans ce processus. Par exemple, la suite du projet EPTiES implique de continuer à analyser et à diffuser les résultats par le biais d'articles, de colloques, etc. Elle se décline aussi par l'intégration de nouveaux partenaires européens (Norvège, Serbie, Grèce, Bulgarie, etc.). Cependant, notre objectif à moyen et à long terme est beaucoup plus pragmatique : nous visons la création et la diffusion de ressources, soit pour une utilisation " libre ", soit pour une intégration dans des systèmes de formation existants. Concrètement, nous envisageons de créer une ressource Web qui servira les besoins des enseignants et des apprenants autant que ceux des chercheurs (observation de pratiques, recensement des besoins, recherche-action).

Notre plus grand souhait est que les enseignants arrivent à consacrer du temps à la prononciation, que leurs efforts soient efficaces pour les apprenants et qu'ils y prennent tous du plaisir en progressant.

\section{Bibliographie}

Bloomfield, L. 1927. « Literate and illiterate speech ». American Speech, vol. 2, $n^{\circ} 10$ : 432-439. DOI : $10.2307 / 451863$

Bloomfield, L. 1933. Language. New York : Henry Holt.

Blum, R. 1999. "Intonation in English, Finnish and French : Ethology versus typology ? ». Actes du colloque du 14e congrès international des sciences phonétiques, ICPhS 99: 301-304.

Boyd, S. 2003. "Foreign-born teachers in the multilingual classroom in Sweden : The role of attitudes to foreign accent ». International Journal of Bilingual Education and Bilingualism, vol. $6, n^{\circ} 3: 283-295$.

DOI : $10.1080 / 13670050308667786$

Bradford, B. \& J. Kenworthy. 1991. " Phonology on teacher training courses ». Speak Out ! vol. 9 : 12-14.

Breitkreutz, J.A., T.M. Derwing \& M.J. Rossiter. 2002. "Pronunciation teaching practices in Canada ». TESL Canada journal, vol. 19 : 51-61.

DOI : 10.18806/tesl.v19i1.919

Burgess, J. \& S. Spencer. 2000. « Phonology and pronunciation in integrated language teaching and teacher education ». System, vol. $28: 191-215$.

DOI : 10.1016/So346-251X(00)00007-5

Conseil de l'Europe. 2001. Cadre européen commun de référence pour les langues : apprendre, enseigner, évaluer. Présentation structurée des échelles du CECR. [En ligne] http://www.coe.int/t/dg4/linguistic/CADRE_FR.asp (consulté le 5 juillet 2012).

Cruttenden, A. (éd.) 2001. Gimson's pronunciation of English (6th edition). London : Routledge.

Davies, A. 2003. The Native speaker : myth and reality. Clevedon : Multilingual Matters.

Derivry-Plard, M. 2008. "Students' representations of native speaker teachers of FL ». In Pawlak, M. (dir.). Investigating English language learning and teaching. Poznań/Kalisz : Faculty of Pedagogy and Fine Arts, Kalisz/Adam Mickiewicz University, 281-293.

Diana, A. 2010. " La phonétique dans l'enseignement de l'anglais aux spécialistes d'autres disciplines : enjeux et priorités ». Cahiers de l'APLIUT, vol. 29, $n^{\circ} 3$ : 10-21.

DOI : $10.4000 /$ apliut.577

Donovan, P.J. 2001. "Making pronunciation a priority for EFL teachers and learners ». In Levey, D., M.A. Losey \& M.A. González (dir). English language teaching changing perspectives in context. Cádiz : Universidad de Cádiz (Servicio de Publicaciones), 245-249.

Dörnyei, Z. \& T. Taguchi. 2010. Questionnaires in second language research construction, administration and processing. London : Routledge.

Dürmüller, U. 2002. "English in Switzerland : From foreign languages to lingua franca ». In Allerton, D., P. Skandera \& C. Tschichold (dir.). Perspectives on English as a world language. Basel : Schwabe, 114-123.

Foote, J.A., A.K. Holtby \& T.M. Derwing. 2011. « Survey of the teaching of pronunciation in adult ESL programs in Canada, 2010 ». TESL Canada journal, vol. 29, $n^{\circ} 1: 1-22$.

Fraser, H. 2001. Teaching pronunciation : A handbook for teachers and trainers ; three frameworks for an integrated approach. Sydney, Australie : New South Wales Department of Education and Training. 
Frost, D. 2011. «Stress and cues to relative prominence in English and French : A perceptual study ». Journal of the International Phonetic Association, vol. 41, $n^{\circ} 1$ : 67-84.

DOI : $10.1017 /$ So025100310000253

Gilbert, J. 2010. «Pronunciation as orphan : What can be done? ». Speak Out !, $43:$ 3-7.

Giles, H., P. Wilson, \& A. Conway. 1981. « Accent and lexical diversity as determinants of impression formation and perceived employment suitability ». Language Sciences, vol. 3, $n^{\circ} 1$ : 91-103.

DOI : 10.1016/So388-0001(81)80015-0

Gonet, W., J. Szpyra-Kozłowska \& R. Święciński. 2010. « Clashes with ashes - the acquisition of vowel reduction by Polish students of English ». In Waniek-Klimczak, E. (dir.). Issues in accents of English 2. Newcastle upon Tyne : Cambridge Scholars Publishing, 213-232.

Henderson, A. 2013. " The English Pronunciation Teaching in Europe Survey (EPTiES) : Initial results \& useful insights for collaborative work ». In Waniek-Klimczak, E. \& L. Shockey (dir.). Teaching and researching English accents in native and non-native speakers. Berlin Heidelberg : Springer Verlag, 123-140.

Hilgendorf, S.K. 2007. « English in Germany : Contact, spread, and attitudes ». World Englishes, vol. $26 n^{\circ} 2: 131-148$.

DOI : 10.1111/j.1467-971X.2007.00498.x

Hirst, D. \& A. Di Cristo (dir.). 1998. Intonation systems : A survey of twenty languages. Cambridge : Cambridge University Press.

Janicka, K., M. Kul \& J. Weckwerth. 2005. « Polish students' attitudes to native English accents as models for ESL pronunciation ». In Dziubalska-Kolaczyk, K. \& J. Przedlacka (dir.). Pronunciation models : A changing scene ? Franckfort-sur-le-Main : Peter Lang Verlag, 251-292.

Kangasvieri, T. et al. 2011. "Kielten tarjonta ja kielivalintojen perusteet perusopetuksessa ». [Language offerings and reasons behind pupils' language study choices in basic education]. Muistiot 2011 : 3. Helsinki : Ministère de l'Éducation Nationale finlandais. [En ligne] http://www.oph.fi/download/138072_Kielten_tarjonta_ja_kielivalintojen_perusteet_perusopetuksessa.pdf (consulté le 5 juillet 2012).

Levey, D. 2001. "Stressing intonation ». In Harris, T. et al. (dir.). ELT2Ooo : thinking back, looking forward. Granada : Greta, 35-45.

Levey, D. 1999. "Half truths and white lies : A practical pronunciation guide for Spanish speakers ». In Harris, T. \& I. Sanz (dir.). ELT : through the looking glass. Granada : Greta, 215226.

Lindemann, S. 2003. "Koreans, Chinese or Indians ? Attitudes and ideologies about non-native English speakers in the United ». Journal of Sociolinguistics, vol. 7, $n^{\circ} 3: 348-364$.

DOI : $10.1111 / 1467-9481.00228$

Lintunen, P. 2004. Pronunciation and phonemic transcription : A study of advanced Finnish learners of English. Turku : University of Turku.

Macdonald, S. 2002. "Pronunciation - views and practices of reluctant teachers ». Prospect, vol. $17, n^{\circ} 3: 3-18$.

Munro, M.J., T.M. Derwing \& K. Sato. 2006. «Salient accents, covert attitudes. Consciousness raising for pre-service second language teachers ». Prospect, vol. 21, $n^{\circ} 1: 67-79$.

Murphy, D. 2011. "An Investigation of English pronunciation teaching in Ireland ». English Today, vol. 27, $n^{\circ} 4: 10-18$.

DOI : $10.1017 /$ So266078411000484

Murphy, J.M. 1997. « Phonology courses offered by MA TESOL programs in the US ». TESOL Quarterly, vol. $31: 741-761$.

Nowacka, M. 2010. "The Ultimate attainment of English pronunciation by Polish college students : A longitudinal study ». In Waniek-Klimczak, E. (dir.). Issues in accents of English 2. Newcastle upon Tyne : Cambridge Scholars Publishing, 233-26o.

Paunović, T. 2009. "Plus ça change... Serbian EFL students' attitudes towards varieties of English ». Poznań Studies in Contemporary Linguistics, vol. 45, $n^{\circ} 4: 511-533$.

DOI : 10.2478/v10010-009-0027-1

Pavon, V. 2001. «El Papel del profesor en la enseñanza de la pronunciación ». In Levey, D., M.A. Losey \& M.A. González (dir.) English language teaching changing perspectives in context. Cádiz : Universidad de Cádiz (Servicio de Publicaciones), 289-300.

Pavon, V. \& A. Rosado. 2003. Guía de fonética y fonología para estudiantes de Filología inglesa en el umbral del siglo XXI. Granada : Comares.

Pawlak, M. (dir.). 2008. Investigating English language learning and teaching. Poznań/Kalisz : Faculty of Pedagogy and Fine Arts, Kalisz/Adam Mickiewicz University.

Penfield, W. \& R. Lamar. 1959. Speech and brain mechanisms. Princeton, NJ : Princeton Univeristy Press.

DOI : $10.1515 / 9781400854677$ 
Sajavaara, K., M. Luukka \& S. Pöyhönen. (2007). " Kielikoulutuspolitiikka suomessa : lähtökohtia, ongelmia ja tulevaisuuden haasteita ». [Language education policy in Finland : basis, problems and future challenges]. In Pöyhönen, S. \& M. Luukka (dir.). Kohti tulevaisuuden kielikoulutusta. Kielikoulutuspoliittisen projektin loppuraportti. Jyväskylä : Jyväskylän yliopisto, Soveltavan kielentutkimuksen keskus, 13-42.

Singleton, D.M. \& L. Ryan. 2004. Language acquisition : the age factor (2nd edition). Clevedon : Multilingual Matters.

Swan, M. \& B. Smith. 2001. Learner English : A teacher's guide to interference and other problems (2nd edition). Cambridge : Cambridge University Press.

Taillefer, G. 2002. " L'anglais dans les formations spécialisées à l'université : un cheveu sur la soupe ? Peut-on rendre le plat plus appétissant ? ». ASp, vol. 37-38 : 155-172.

DOI : $10.4000 /$ asp.1531

Tay, M.W. 1982. "The Phonology of educated Singaporean English ». English Worldwide, vol. $3: 135-145$.

Tergujeff, E., R. Ullakonoja \& H. Dufva. 2011. « Phonetics and foreign language teaching in Finland ». In Werner, S. \& T. Kinnunen (eds.). XXVI Fonetiikan päivät 201O. Joensuu, Finland : University of Eastern Finland, 63-68.

Truchot, C. 2002. L'anglais en Europe : repères. Strasbourg : Conseil de l'Europe.

Walker, R. 1999. "Proclaimed and perceived wants and needs among Spanish teachers of English ». Speak Out !, vol. 24 : 25-32.

Waniek-Klimczak, E. \& K. Klimczak. 2005. « Target in speech development : learners' views ». In Dziubalska-Kołaczyk, K. \& J. Przedlacka (dir.). Pronunciation models : A changing scene ? Francfort-sur-le-Main : Peter Lang Verlag, 229-250.

\section{Notes}

1 « Greater linguistic competence is characterized by a better 'accent or pronunciation' ». (Les traductions des citations en anglais sont des auteurs de l'article).

2 «Some strong social schema is in play mystifying the speaker and the teacher, the native speaker and the native teacher. "

3 Davies cherche à démêler les mythes et les réalités du concept du « natif », surtout par rapport aux questions d'identité et de pouvoir. Il pose les questions suivantes : "...the relevant questions of the relation between being a native speaker and being a second language learner, raising the question of whether the latter can become the former » (2003:6).

4 Par exemple, en 1927 Bloomfield parle déjà de " the native language that one learned upon one's mother's knee » (1927: 151) et, six ans plus tard, il énonce plus explicitement : " the first language a human being learns to speak» (1933 : 43). Voir aussi le texte original du Critical Age Hypothesis (Penfield \& Lamar 1959) ainsi que l'analyse critique de cette notion par Singleton \& Ryan (2004).

5 Selon Tay, un natif est « one who learns English in childhood and continues to use it as his/her dominant language which has reached a certain level of fluency » (1982 : 67-68).

6 Sur 65 répondants, 39 indiquent avoir le CAPES et 18 l'Agrégation, avec 5 participants ayant les deux concours.

7 C'est-à-dire qu'ils ont réussi le CAPES et/ou l'Agrégation, les deux concours de recrutement de professeurs en France. Depuis 2011, on ne peut se présenter au CAPES que dans l'année de Master 2 et, pour se présenter à l'Agrégation, il faut être titulaire d'un Master 2.

8 Voir, par exemple, le manuel de formation de Fraser (2001) ou encore les suggestions de Gilbert (2010).

9 CELTA : Certificate in teaching English to speakers of other languages (un diplôme qui se prépare en un mois intensif, ou sur une durée plus longue à distance, et qui forme les anglophones natifs titulaires d'une Licence à enseigner l'anglais à l'étranger).

10 PGCE : Post-graduate certificate of education (une année diplômante et professionnalisante suivant le Bachelor's au Royaume-Uni).

11 Voir Truchot pour les statistiques par rapport au nombre de thèses de doctorat en anglais : " En Suisse, pays où sont utilisées pourtant des langues de plus grande diffusion, c'est un choix qui est fait de plus en plus souvent. » (2002:11).

12 «I'm very conscious that I know absolutely nothing about phonetics ».

13 «Bad pronunciation is chronic throughout the French academic system ».

\section{Table des illustrations}




\begin{tabular}{|c|c|c|}
\hline 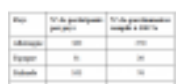 & Titre & $\begin{array}{l}\text { Tableau 1. Participants par pays, nombre total de participants et nombre } \\
\text { de questionnaires remplis à } 100 \% \text { par pays }\end{array}$ \\
\hline & UيLي & http://journals.openedition.org/apliut/docannexe/image/3586/img-1.png \\
\hline & Fichier & image/png, 28k \\
\hline & Titre & Tableau 2. Quelques données sur les participants \\
\hline & URI & http://journals.openedition.org/apliut/docannexe/image/3586/img-2.png \\
\hline & Fichier & image/png, 26k \\
\hline & Titre & $\begin{array}{l}\text { Fig. 1. Réponses à l'item } 60 \text { (l'importance de l'anglais par rapport à } \\
\text { d'autres langues) }\end{array}$ \\
\hline & Légende & $1=$ « pas important du tout $», 5=$ « très important $»$ \\
\hline & URL & http://journals.openedition.org/apliut/docannexe/image/3586/img-3.png \\
\hline & Fichier & image/png, 32k \\
\hline & Titre & $\begin{array}{l}\text { Fig. 2. Réponses à l'item } 61 \text { (l'importance de la prononciation par } \\
\text { rapport à d'autres compétences langagières) }\end{array}$ \\
\hline & Lég & $1=$ « pas important du tout $», 5=$ « très important » \\
\hline & UيL & http://journals.openedition.org/apliut/docannexe/image/3586/img-4.png \\
\hline & ier & image/png, 29k \\
\hline & & $\begin{array}{l}\text { Fig. 3. Réponses à l'item } 63 \text { (Comment estimez-vous votre propre } \\
\text { prononciation?) }\end{array}$ \\
\hline & Légende & $1=$ « extrêmement mauvaise », $5=$ " excellente » \\
\hline & UيLي & http://journals.openedition.org/apliut/docannexe/image/3586/img-5.png \\
\hline & Fichier & image/png, 35k \\
\hline & Titre & $\begin{array}{l}\text { Fig. } 4 \text {. Réponses à l'item } 57 \text { (la qualité de la formation en prononciation } \\
\text { reçue) }\end{array}$ \\
\hline & Légende & $1=$ « très mauvaise $"$ et $5=$ « excellente » \\
\hline & URL & http://journals.openedition.org/apliut/docannexe/image/3586/img-6.png \\
\hline & ier & image/png, 31k \\
\hline & Titre & $\begin{array}{l}\text { Fig. 5. Réponses à l'item } 64 \text { sur la conscience des objectifs des } \\
\text { apprenants }\end{array}$ \\
\hline & URي & http://journals.openedition.org/apliut/docannexe/image/3586/img-7.png \\
\hline & Fichier & image/png, 46k \\
\hline & Titre & $\begin{array}{l}\text { Fig. 6. Réponses à l'item } 65 \text { sur la conscience des compétences des } \\
\text { apprenants }\end{array}$ \\
\hline & URي & http://journals.openedition.org/apliut/docannexe/image/3586/img-8.png \\
\hline & Fic & image/png, 46k \\
\hline & Titre & $\begin{array}{l}\text { Fig. } 7 \text {. Réponses à l'item } 66 \text { sur le degré de motivation des apprenants } \\
\text { pour parler anglais }\end{array}$ \\
\hline & UيLي & http://journals.openedition.org/apliut/docannexe/image/3586/img-9.png \\
\hline & Fichier & image/png, 33k \\
\hline & Titre & $\begin{array}{l}\text { Fig. 8. Réponses à l'item } 67 \text { sur le désir des apprenants de parler } \\
\text { anglais comme un natif }\end{array}$ \\
\hline & & http://journals.openedition.org/apliut/docannexe/image/3586/img-10.png \\
\hline & Fichier & image/png, 49k \\
\hline
\end{tabular}

\section{Pour citer cet article}

Référence papier

Dan Frost et Alice Henderson, « Résultats du sondage EPTiES (English Pronunciation Teaching in Europe Survey) : l'enseignement de la prononciation dans plusieurs pays européens vu par les enseignants ", Recherche et pratiques pédagogiques en langues de spécialité, Vol. XXXII N 1 | 2013, 92-113.

Référence électronique

Dan Frost et Alice Henderson, « Résultats du sondage EPTiES (English Pronunciation Teaching in Europe Survey) : l'enseignement de la prononciation dans plusieurs pays européens vu par les enseignants », Recherche et pratiques pédagogiques en langues de spécialité [En ligne], Vol. XXXII N 1 | 2013, mis en ligne le 18 février 2013, consulté le 20 décembre 2018. URL: http://journals.openedition.org/apliut/3586 ; DOI : 10.4000/apliut.3586 


\section{Cet article est cité par}

- Henderson, Alice. Jarosz, Anna. (2014) Desperately Seeking a Communicative Approach: English Pronunciation in a Sample of French and Polish Secondary School Textbooks. Research in Language, 12. DOI: 10.2478/rela-2014-0015

\section{Auteurs}

\section{Dan Frost}

Dan Frost est Maître de conférences en anglais à l'Université de Savoie à Chambéry où il enseigne au département LEA. II enseigne l'anglais depuis 1993 et exerce en France depuis 1996. Avant de travailler à l'Université de Savoie, il a enseigné pendant deux ans en lycée et pendant dix ans à l'IUT de Grenoble. Ses recherches portent sur la didactique de l'anglais oral, l'enseignement et l'apprentissage de la phonologie et de la prononciation de l'anglais ainsi que sur l'apprentissage médié par ordinateur. dan.frost@univ-savoie.fr

Articles du même auteur

Introduction [Texte intégral]

Paru dans Recherche et pratiques pédagogiques en langues de spécialité, Vol. 37 №2 | 2018

L'innovation est le ton qui fait la chanson : une approche musico-prosodique en secteur Lansad [Texte intégral]

Paru dans Recherche et pratiques pédagogiques en langues de spécialité, Vol. 35 Nº spécial 1 | 2016

Success: B2 or not B2- that is the question [Texte intégral]

(The Ello Project - Étude longitudinale sur la langue orale)

Paru dans Recherche et pratiques pédagogiques en langues de spécialité, Vol. XXXIV Nº 2 | 2015

Alain Marchive, La pédagogie à l'épreuve de la didactique. Approche historique, perspectives théoriques et recherches empiriques [Texte intégral]

Presses Universitaires de Rennes, 2008.

Paru dans Cahiers de l'APLIUT, Vol. XXVIII N 3 | 2009

Nicolas Guichon, Langues et TICE. Méthodologie de conception multimédia [Texte intégral]

Paris : Ophrys, 2006

Paru dans Cahiers de l'APLIUT, Vol. XXVI N $1 \mid 2007$

Seeing is Believing : l'oscillogramme, l'auto-apprentissage et l'accentuation de mots [Texte intégral]

Paru dans Recherche et pratiques pédagogiques en langues de spécialité, Vol. XXI N 3 | 2002

Tous les textes...

Alice Henderson

Alice Henderson est Maître de conférences en anglais à l'Université de Savoie à Chambéry où, depuis 18 ans, elle enseigne aux étudiants de LCE et du secteur LANSAD en sciences humaines. Elle est intervenue dans la formation des enseignants en Pologne, en Espagne, en Norvège et aux USA. Ses domaines de recherche sont : la didactique de l'anglais oral, l'enseignement et l'apprentissage de la phonologie de l'anglais, ainsi que la politique linguistique. alice.henderson@univ-savoie.fr

\section{Droits d'auteur}

Association des Professeurs de Langues des Instituts Universitaires de Technologie 